\title{
ON A CLASS OF MONOMIAL IDEALS
}

\author{
KEIVAN BORNA ${ }^{\bowtie}$ and RAHELEH JAFARI
}

(Received 22 May 2012; accepted 26 October 2012; first published online 28 January 2013)

\begin{abstract}
Let $S$ be a polynomial ring over a field $K$ and let $I$ be a monomial ideal of $S$. We say that $I$ is MHC (that is, $I$ satisfies the maximal height condition for the associated primes of $I$ ) if there exists a prime ideal $p \in \operatorname{Ass}_{S} S / I$ for which ht(p) equals the number of indeterminates that appear in the minimal set of monomials generating $I$. Let $I=\bigcap_{i=1}^{k} Q_{i}$ be the irreducible decomposition of $I$ and let $m(I)=\max \left\{\left|Q_{i}\right|-\operatorname{ht}\left(Q_{i}\right): 1 \leq i \leq k\right\}$, where $\left|Q_{i}\right|$ denotes the total degree of $Q_{i}$. Then it can be seen that when $I$ is primary, $\operatorname{reg}(S / I)=m(I)$. In this paper we improve this result and show that whenever $I$ is MHC, then $\operatorname{reg}(S / I)=m(I)$ provided $\left|\operatorname{Ass}_{S} S / I\right| \leq 2$. We also prove that $m\left(I^{n}\right) \leq n \max \left\{\left|Q_{i}\right|: 1 \leq i \leq k\right\}-\operatorname{ht}(I)$, for all $n \geq 1$. In addition we show that if $I$ is MHC and $w$ is an indeterminate which is not in the monomials generating $I$, then $\operatorname{reg}\left(S /\left(I+w^{d} S\right)^{n}\right) \leq \operatorname{reg}(S / I)+n d-1$ for all $n \geq 1$ and $d$ large enough. Finally, we implement an algorithm for the computation of $m(I)$.
\end{abstract}

2010 Mathematics subject classification: primary 13P20; secondary 13D02, 68W30, 13D45.

Keywords and phrases: associated primes, Castelnuovo-Mumford regularity, powers of ideals, primary ideals, local cohomology.

\section{Introduction}

Throughout this paper $S=K\left[x_{1}, \ldots, x_{s}\right]$ is a polynomial ring over a field $K$. Then $S$ is a *local graded ring with *maximal ideal $m=S_{+}$. The Castelnuovo-Mumford regularity, or simply regularity, of a graded ideal $I$ is an invariant which is related to free resolutions, and thus it measures the complexity of $I$. More precisely,

$$
\operatorname{reg}(S / I)=\max \left\{e_{i j}-i: 0 \leq i \leq p, 1 \leq j \leq \beta_{i}\right\}+1,
$$

where

$$
0 \longrightarrow \bigoplus_{j=1}^{\beta_{p}} S\left(-e_{p j}\right) \longrightarrow \cdots \longrightarrow \bigoplus_{j=1}^{\beta_{0}} S\left(-e_{0 j}\right) \longrightarrow I \longrightarrow 0
$$

is a minimal graded free resolution of $I$. Regularity is also defined in terms of local cohomology. Let $n=\operatorname{dim}(S / I)$ and $\operatorname{end}\left(\mathrm{H}_{\mathrm{m}}^{i}(S / I)\right)=\max \left\{t: \mathrm{H}_{\mathrm{m}}^{i}(S / I)_{t} \neq 0,0 \leq i \leq n\right\}$, where $\mathrm{H}_{\mathrm{m}}^{i}(S / I)$ is the $i$ th local cohomology module with the support in $\mathrm{m}$. Then

The first author is grateful to the National Elite Foundation of Iran for financial support. The research of the second author was supported in part by a grant from IPM (No. 91130020).

(C) 2013 Australian Mathematical Publishing Association Inc. 0004-9727/2013 \$16.00 
the regularity of $S / I$ is the number $\operatorname{reg}(S / I)=\max \left\{\operatorname{end}\left(\mathrm{H}_{\mathrm{m}}^{i}(S / I)\right)+i: 0 \leq i \leq n\right\}$; see $[4,9]$ for more information.

The problem of regularity of powers of an ideal has been studied by many authors; see for example [1, 3, 5, 7, 8, 12-15]. More specially, Cutkosky et al. [8] and Kodiyalam [14] studied the asymptotic behaviour of the regularity and independently showed that the regularity of $I^{k}$ is a linear function for large $k$, that is, $\operatorname{reg}\left(I^{k}\right)=$ $a(I) k+b(I)$, for all $k \geq c(I)$. Note also that by [8, Theorem 1.1] there exists a constant integer $e$ such that, for all $n \geq 1$,

$$
\operatorname{reg}\left(S / I^{n}\right) \leq n d(I)+e .
$$

This problem for the special family of monomial ideals is interesting as well. For instance, in [13] Hoa and Trung found some relationships between the regularity and arithmetic degree of monomial ideals. They also studied the problem of finding upper bounds for the regularity of monomial ideals. More precisely, in [13, Theorem 3.4] they proved that, for a monomial ideal $I$ of $S$ with minimal monomial generating set $\left\{m_{1}, \ldots, m_{s}\right\}$ for which $\operatorname{deg}\left(m_{1}\right) \geq \cdots \geq \operatorname{deg}\left(m_{s}\right)$, then $\operatorname{reg}(S / I) \leq \operatorname{deg}\left(m_{1}\right)+\cdots+$ $\operatorname{deg}\left(m_{u}\right)-u$, where $u=\min \{s, n\}$. Furthermore, equality holds if and only if $I$ is a complete intersection.

In this paper we study the associated primes and regularity of monomial ideals. For a monomial ideal $I$ of a polynomial ring $S$, we say that $I$ is $\mathrm{MHC}$ (that is, $I$ satisfies the maximal height condition for the associated primes of $I$; see Definition 2.3) if there exists a prime ideal $\mathfrak{p} \in \operatorname{Ass}_{S} S / I$ for which ht(p) equals the number of indeterminates that appear in the minimal set of monomials generating $I$. Let $I=\bigcap_{i=1}^{k} Q_{i}$ be the irreducible decomposition of $I$. We define $m(I)=\max \left\{\left|Q_{i}\right|-\operatorname{ht}\left(Q_{i}\right): 1 \leq i \leq k\right\}$, where $|Q|$ denotes the total degree of $Q$. We prove that if $I$ is an MHC monomial ideal for which $\left|\operatorname{Ass}_{S} S / I\right| \leq 2$, then $\operatorname{reg}(S / I)=m(I)$; see Theorem 3.3. We also prove that $m\left(I^{n}\right) \leq n \max \left\{\left|Q_{i}\right|: 1 \leq i \leq k\right\}-\operatorname{ht}(I)$ for all $n \geq 1$; see Proposition 2.10.

It is easy to give examples of ideals that are not MHC but have certain powers which are so; see Example 2.11. We show that all powers of the square-free Veronese ideals are MHC; see Example 2.6.

Let $\Lambda(I)=\left\{x_{1}, \ldots, x_{t}\right\}$ be the set of indeterminates that appear in the minimal set of monomials generating $I$ and $S^{\prime}=K\left[x_{1}, \ldots, x_{t}\right]$. Note that the definition of MHC depends on $\Lambda(I)$. It is now natural to ask whether $I$ remains MHC when we restrict the base ring to $S^{\prime}$. In Proposition 2.9 we provide a positive answer and show that $\mathfrak{p} \in \operatorname{Ass}_{S} S / I$ if and only if $\mathfrak{p} \in \operatorname{Ass}_{S^{\prime}} S^{\prime} / I S^{\prime}$, where $\mathfrak{p}=\left(x_{1}, \ldots, x_{t}\right)$.

Finally, we prove that for a monomial ideal $I$ which is MHC, if $w$ is an indeterminate which is not in the monomials generating $I$ and $d \geq \operatorname{reg}(I)+e$, where $e$ is proposed as in (1.1), then $\operatorname{reg}\left(S /\left(I+w^{d} S\right)^{n}\right) \leq \operatorname{reg}(S / I)+n d-1$, for all $n \geq 1$; see Proposition 3.6.

The organisation of this paper is as follows. In Section 2 we deal with some definitions, examples and applications of ideals that are MHC. Section 3 is devoted to the regularity of monomial ideals and their powers. Finally, some implementations to compute $m(I)$ are presented in Appendix A. 


\section{The maximal height condition for the associated primes of $I$}

Let $I$ be a monomial ideal of $S$. Then $I$ has a unique minimal set of monomial generators, $G(I)$; see [11, Proposition 1.1.6]. It is common to denote the cardinality of $G(I)$ by $\mu(I)$. If $G(I)=\left\{u_{1}, \ldots, u_{r}\right\}$, we define $d(I)=\max \left\{\operatorname{deg}\left(u_{i}\right): 1 \leq i \leq r\right\}$. Assume that $\operatorname{lcm}\left(u_{1}, \ldots, u_{r}\right)=x_{i_{1}}^{a_{1}} \ldots x_{i_{t}}^{a_{t}}$, where $a_{j}>0$ for $j=1, \ldots, t$. Then we denote $t$ by $\lambda(I)$ and set $\Lambda(I)=\left\{x_{i_{1}}, \ldots, x_{i_{t}}\right\}$. Indeed $\lambda(I)$ is the number of indeterminates that appear in the minimal set of monomial generators of $I$ and, as mentioned in the following, $\operatorname{ht}(\mathfrak{p}) \leq \lambda(I)$ for all $\mathfrak{p} \in \operatorname{Ass}_{S} S / I$. If $I=\left(x_{i_{1}}^{a_{1}}, \ldots, x_{i_{t}}^{a_{t}}\right)$, then we denote the total degree of $I$ by $|I|$, that is, $|I|=\sum_{j=1}^{t} a_{j}$.

We first recall the following theorem due to Vasconcelos, which has an essential role in the paper.

Theorem 2.1 [11, Theorem 1.3.1, Proposition 1.3.5]. Let I be a monomial ideal of $S$. Then there is an irreducible decomposition $I=\bigcap_{i=1}^{k} Q_{i}$, where $Q_{i}$ is of the form $\left(x_{i_{1}}^{a_{1}}, x_{i_{2}}^{a_{2}}, \ldots, x_{i_{t}}^{a_{t}}\right)$ which is $\mathfrak{p}_{i}:=\left(x_{i_{1}}, \ldots, x_{i_{t}}\right)$-primary. Moreover, this decomposition is unique.

Sketch of PRoof. Assume that $I$ is a monomial ideal, $G(I)=\left(u_{1}, \ldots, u_{r}\right)$ and that $u_{1}$ is not a pure power. Then we can write $u_{1}=v w$ where $v$ and $w$ are coprime monomials, that is, $\operatorname{gcd}(v, w)=1, v \neq 1$ and $w \neq 1$. One can see that $I=I_{1} \cap I_{2}$ where $I_{1}=\left(v, u_{2}, \ldots, u_{r}\right)$ and $I_{2}=\left(w, u_{2}, \ldots, u_{r}\right)$. If either of $G\left(I_{1}\right)$ or $G\left(I_{2}\right)$ contains an element which is not a pure power, we proceed as before and obtain after a finite number of steps a presentation $I=Q_{1} \cap \cdots \cap Q_{k}$ as an intersection of monomial ideals generated by pure powers.

The irreducible decomposition $I=\bigcap_{i=1}^{k} Q_{i}$ is fixed throughout this paper and will be used hereafter.

Remark 2.2. Assume that $I=\bigcap_{i=1}^{k} Q_{i}$ is the irreducible decomposition of $I$. Since any indeterminate which appears in $G\left(Q_{j}\right)$ also appears in $G(I)$, we have $\lambda\left(Q_{j}\right) \leq \lambda(I)$ for all $1 \leq j \leq k$. Let $\mathfrak{p} \in \mathrm{Ass}_{S} S / I$. Then Theorem 2.1 implies that there is some $j$, $1 \leq j \leq k$, such that $Q_{j}$ is a p-primary ideal. Thus ht $(\mathfrak{p})=\lambda\left(Q_{j}\right) \leq \lambda(I)$.

It now makes perfect sense to ask when $\mathrm{ht}(\mathfrak{p})=\lambda(I)$ for some $\mathfrak{p} \in \operatorname{Ass}_{S} S / I$.

Definition 2.3. Assume that $I$ is a monomial ideal of $S$. We say that $I$ is MHC (that is, $I$ satisfies the Maximal Height Condition for the associated primes of $I$ ) if there exists a prime ideal $\mathfrak{p} \in \mathrm{Ass}_{S} S / I$ such that $h t(\mathfrak{p})=\lambda(I)$.

REMARK 2.4. If a monomial ideal $I$ of $S$ is MHC, then $\lambda(I) \leq \mu(I)$. In fact $\lambda(I)=$ $\operatorname{ht}\left(Q_{j}\right)=\mu\left(Q_{j}\right)$ for some $1 \leq j \leq k$. On the other hand, the proof of Theorem 2.1 implies that $\mu\left(Q_{j}\right) \leq \mu(I)$.

Remark 2.5. Let $\Lambda(I)=\left\{x_{1}, \ldots, x_{t}\right\}$. Then

$I$ is MHC if and only if depth $\left((S / I)_{\left(x_{1}, \ldots, x_{t}\right)}\right)=0$. 
To see this, note that

$$
\begin{aligned}
I \text { is } \mathrm{MHC} & \Longleftrightarrow\left(x_{1}, \ldots, x_{t}\right) \in \operatorname{Ass}_{S} S / I \\
& \Longleftrightarrow\left(x_{1}, \ldots, x_{t}\right) S_{\left(x_{1}, \ldots, x_{t}\right)} \in \operatorname{Ass}_{S}(S / I)_{\left(x_{1}, \ldots, x_{t}\right)} \\
& \Longleftrightarrow \operatorname{depth}\left((S / I)_{\left(x_{1}, \ldots, x_{t}\right)}\right)=0 .
\end{aligned}
$$

Now in conjunction with [10, Corollary 3.4] we give an ideal for which all of its large powers are MHC. Recall that the square-free Veronese ideal of degree $d$ in the variables $x_{i_{1}}, \ldots, x_{i_{t}}$ is the ideal of $S$ which is generated by all square-free monomials in $x_{i_{1}}, \ldots, x_{i_{t}}$ of degree $d$. Further, each power $I^{k}$ is a Veronese ideal of degree $k d$.

ExAmple 2.6. Let $2 \leq d<s$ and let $I=I_{s, d}$ be the square-free Veronese ideals of degree $d$ in the variables $x_{1}, \ldots, x_{s}$. Then depth $\left(S / I^{k}\right)=\max \{0, s-k(s-d)-1\}$. Hence, for $k$ large enough we have depth $\left(S / I^{k}\right)=0$ and now Remark 2.5 implies that $I^{k}$ are MHC.

Definition 2.7. Assume that $I$ is a monomial ideal of $S$. We define

$$
m(I)=\max \left\{\left|Q_{j}\right|-\operatorname{ht}\left(Q_{j}\right): 1 \leq j \leq k\right\} .
$$

LeMma 2.8. Assume that $I$ and $J$ are monomial ideals and $u=x_{1}^{a_{1}} \cdots x_{s}^{a_{s}}$, where $a_{i} \geq 0$ for each $1 \leq i \leq s$. Let $\Lambda(I)=\left\{x_{1}, \ldots, x_{t}\right\}$. Then the following results hold.

(i) $\quad(I \cap J) u=I u \cap J u$.

(ii) $m(I \cap J) \leq \max \{m(I), m(J)\}$.

(iii) $m(I u)=\max \left\{m(I)+\sum_{i=1}^{t} a_{i}, a_{t+1}-1, \ldots, a_{s}-1\right\}$.

(iv) $m(J)=m(I)+a_{t+1}-1$, where $J=I+x_{t+1}^{a_{t+1}} S$.

Proof. Let $G(I)=\left\{u_{1}, \ldots, u_{m}\right\}$ and $G(J)=\left\{v_{1}, \ldots, v_{n}\right\}$.

(i) By [11, Proposition 1.2.1], $I \cap J=\left\langle\operatorname{lcm}_{i, j}\left(u_{i}, v_{j}\right)\right\rangle$. Thus

$$
I u \cap J u=\left\langle\operatorname{lcm}_{i, j}\left(u_{i} u, v_{j} u\right)\right\rangle=\left\langle\operatorname{lcm}_{i, j}\left(u_{i}, v_{j}\right) u\right\rangle=\left\langle\operatorname{lcm}_{i, j}\left(u_{i}, v_{j}\right)\right\rangle u=(I \cap J) u .
$$

(ii) Let $I=\bigcap_{i=1}^{n} Q_{i}$ and $J=\bigcap_{i=n+1}^{m} Q_{i}$ be the irreducible decompositions of $I$ and $J$, respectively. Then

$$
I \cap J=\left(\bigcap_{i=1}^{n} Q_{i}\right) \cap\left(\bigcap_{i=n+1}^{m} Q_{i}\right) .
$$

Thus $m(I \cap J) \leq \max \{m(I), m(J)\}$.

(iii) Let $I^{\prime}=I\left(x_{1}^{a_{1}} \cdots x_{t}^{a_{t}}\right)$. It is now easy to see that

$$
m\left(I^{\prime}\right)=m(I)+\sum_{i=1}^{t} a_{i}
$$

and

$$
m(I u)=m\left(I^{\prime}\left(x_{t+1}^{a_{t+1}} \cdots x_{s}^{a_{s}}\right)\right)=\max \left\{m\left(I^{\prime}\right): a_{t+1}-1, \ldots, a_{s}-1\right\} .
$$

(iv) Since $J=\bigcap_{i=1}^{t}\left(Q_{i}+x_{t+1}^{a_{t+1}} S\right)$ is the irreducible decomposition of $J$, the total degree of each component increases by $a_{t+1}$ and their height grows only by one. Now if $m(I)=\left|Q_{1}\right|-\operatorname{ht}\left(Q_{1}\right)$, then $m(J)=\left|Q_{1}^{\prime}\right|-\operatorname{ht}\left(Q_{1}^{\prime}\right)$ where $Q_{1}^{\prime}:=Q_{1}+x_{t+1}^{a_{t+1}} S$. Thus $m(J)=m(I)+a_{t+1}-1$. 
In the following proposition we show that, concerning the MHC property, the base ring can be restricted to a special subring of it.

Proposition 2.9. Let $I$ be a monomial ideal of $S=K\left[x_{1}, \ldots, x_{s}\right]$ with $\Lambda(I)=$ $\left\{x_{1}, \ldots, x_{t}\right\}$ and let $S^{\prime}=K\left[x_{1}, \ldots, x_{t}\right]$ and $\mathfrak{p}=\left(x_{1}, \ldots, x_{t}\right)$. Then the following statements are equivalent:

(i) $\mathfrak{p} \in \operatorname{Ass}_{S} S / I$;

(ii) $\mathfrak{p} \in \operatorname{ass}_{S^{\prime}} S^{\prime} / I S^{\prime}$.

In particular, I is $M H C$ if and only if $I S^{\prime}$ is $M H C$.

Proof. (ii) $\Longrightarrow$ (i) This is easily seen from the monomorphism $S^{\prime} / I S^{\prime} \hookrightarrow S / I$.

(i) $\Longrightarrow$ (ii) Let $\mathfrak{p} \in \operatorname{Ass}_{S} S / I$. Then there exist some $u \notin I$ such that $\mathfrak{p}=I: u$. Thus $I: \mathfrak{p} \nsubseteq I$. Since $I: \mathfrak{p}$ is monomial by [11, Proposition 1.2.2], we can assume that $u$ is monomial. Let $u=x_{1}^{a_{1}} \cdots x_{s}^{a_{s}}$, where $a_{i} \geq 0$ for $1 \leq i \leq s$. We claim that $u^{\prime}=x_{1}^{a_{1}} \cdots x_{t}^{a_{t}} \in(I: \mathfrak{p}) \backslash I$, from which it follows that $\mathfrak{p} \subseteq I: u^{\prime} \subseteq I: u=\mathfrak{p}$ and thus the result follows. To prove the claim let $1 \leq i \leq t$ and note that $u x_{i} \in I$ so that $v \mid u x_{i}$ for some $v \in G(I)$. Thus

$$
u x_{i}=\alpha v \quad \text { for some } \alpha \in S .
$$

Let $u^{\prime \prime}=x_{t+1}^{a_{t+1}} \cdots x_{s}^{a_{s}}$. Then $u^{\prime \prime} \mid \alpha v$ by (2.1) and since $\operatorname{gcd}\left(u^{\prime \prime}, v\right)=1$ it follows that $u^{\prime \prime} \mid \alpha$, that is,

$$
\alpha=u^{\prime \prime} \beta \quad \text { for some } \beta \in S .
$$

Now (2.1) and (2.2) imply that $\beta v=u^{\prime} x_{i}$. Hence $u^{\prime} \mathfrak{p} \subseteq I$, that is, $u^{\prime} \in I: \mathfrak{p}$. Finally, since $u \notin I$ it is clear that $u^{\prime} \notin I$.

In the following proposition we obtain an upper bound for $m\left(I^{n}\right)$ in terms of ht $(I)$ and the irreducible decomposition of $I=\bigcap_{i=1}^{k} Q_{i}$.

Proposition 2.10. Let I be a monomial ideal of $S$. Then, for all $n \geq 1$,

$$
m\left(I^{n}\right) \leq n \max \left\{\left|Q_{i}\right|: 1 \leq i \leq k\right\}-\operatorname{ht}(I)
$$

Proof. Let $\Lambda(I)=\left\{x_{1}, \ldots, x_{t}\right\}$ and $n \geq 1$ be an integer. Notice that $\lambda\left(I^{n}\right)=\lambda(I)$. Let $Q_{1}=\left(x_{1}^{a_{1}}, \ldots, x_{s}^{a_{s}}\right)$ for which $\left|Q_{1}\right|=\max \left\{\left|Q_{i}\right|: 1 \leq i \leq k\right\}$. Furthermore assume that $I^{n}=\bigcap_{j=1}^{m} Q_{j}^{\prime}$ is the irreducible primary decomposition of $I^{n}$. Now it is easy to see that $\left|Q_{j}^{\prime}\right| \leq n\left|Q_{1}\right|$. Finally, we conclude that

$$
\begin{aligned}
m\left(I^{n}\right) & =\max \left\{\left|Q_{j}^{\prime}\right|-\operatorname{ht}\left(Q_{j}^{\prime}\right): 1 \leq j \leq m\right\} \leq n\left|Q_{1}\right|-\operatorname{ht}(I) \\
& =n \max \left\{\left|Q_{i}\right|: 1 \leq i \leq k\right\}-\operatorname{ht}(I) .
\end{aligned}
$$

This concludes the proof.

Note that $\lambda(I)=\lambda\left(I^{n}\right)$ for all $n \geq 1$. We now give an example in which $I$ is not MHC but $I^{2}$ and $I^{3}$ are MHC. 
Example 2.11. Let $S=K[x, y, z]$ and $I=(x y, z)=(x, z) \cap(y, z)$. Then $\lambda(I)=3$ and $(x, y, z) \notin \operatorname{Ass}_{S} S / I$. Thus $I$ is not MHC. On the other hand,

$$
I^{2}=\left(x^{2} y^{2}, x y z, z^{2}\right)=\left(x^{2}, x y z, z^{2}\right) \cap\left(y^{2}, x y z, z^{2}\right)=Q_{1} \cap \cdots \cap Q_{6},
$$

where

$$
\begin{gathered}
Q_{1}=\left(x, z^{2}\right), \quad Q_{2}=\left(x^{2}, z\right), \quad Q_{3}=\left(y, z^{2}\right), \quad Q_{4}=\left(y^{2}, z\right), \\
Q_{5}=\left(x^{2}, y, z^{2}\right) \quad \text { and } \quad Q_{6}=\left(x, y^{2}, z^{2}\right) .
\end{gathered}
$$

Note that $Q_{1}, \ldots, Q_{4}$ are primary ideals of height 2 and $Q_{5}, Q_{6}$ are $(x, y, z)$-primary ideals of height 3 . Note also that $\left|Q_{i}\right|=3$ for $i \in\{1,2,3,4\}$ and $\left|Q_{i}\right|=5$ for $i \in\{5,6\}$. Thus $I^{2}$ is MHC and $m\left(I^{2}\right)=\max \{5-3,3-2\}=2$. Furthermore, the irreducible decomposition of $I^{3}$ is $I^{3}=Q_{1} \cap \cdots \cap Q_{7}$, where

$$
\begin{gathered}
Q_{1}=\left(x, z^{3}\right), \quad Q_{2}=\left(x^{3}, z\right), \quad Q_{3}=\left(x^{2}, z^{2}\right), \quad Q_{4}=\left(x^{2}, y, z^{3}\right), \quad Q_{5}=\left(x^{3}, y, z^{3}\right), \\
Q_{6}=\left(x^{3}, y^{2}, z^{2}\right) \quad \text { and } \quad Q_{7}=\left(x, y^{3}, z^{3}\right) .
\end{gathered}
$$

Hence $I^{3}$ is MHC. Note that $\left|Q_{i}\right|=4$ for $i \in\{1,2,3\},\left|Q_{4}\right|=6$ and $\left|Q_{i}\right|=7$ for $i \in$ $\{5,6,7\}$. Thus $m\left(I^{3}\right)=\max \{4-2,6-3,7-3\}=4$.

ExAmple 2.12. Let $S=K[x, y, z]$ and $I=\left(x^{2}, x y, y^{10}, x z\right)=\left(x^{2}, y, z\right) \cap\left(x, y^{10}\right)$. Then $I$ is MHC and $m(I)=\max \{4-3,11-2\}=9$. Furthermore, $\operatorname{reg}(S / I)=9$, see [6]. In Theorem 3.3 we show that this phenomenon happens for all ideals that are MHC and satisfy $\left|\operatorname{Ass}_{S} S / I\right| \leq 2$.

\section{Regularity of powers of monomial ideals that are $\mathrm{MHC}$}

We begin this section with the following well-known result.

Remark 3.1. Let $I=\left(x_{1}^{\alpha_{1}} \cdots x_{s}^{\alpha_{s}}\right)$. Then $I$ is a complete intersection and so by [13, Theorem 3.4] we have $\operatorname{reg}(S / I)=\sum_{i=1}^{s} \alpha_{i}-1=m(I)$.

In the following two results we generalise the formula of Remark 3.1 to primary monomial ideals and monomial ideals that are MHC.

Lemma 3.2. Let I be a primary monomial ideal of $S$. Then $\operatorname{reg}(S / I)=m(I)$.

Proof. Let $I=\bigcap_{j=1}^{k} Q_{j}$ be the irreducible decomposition of $I$. If $I$ is $m$-primary, then for a fixed $1 \leq j \leq k$, let $Q_{j}=\left(x_{1}^{a_{1}}, \ldots, x_{s}^{a_{s}}\right)$ for some positive integers $a_{1}, \ldots, a_{s}$. Then it is easy to see that each monomial of degree exceeding $\sum_{i=1}^{s} a_{i}-s$ is in $Q_{j}$ and $x_{1}^{a_{1}-1} \cdots x_{s}^{a_{s}-1} \notin Q_{j}$. Thus

$$
\operatorname{end}\left(S / Q_{j}\right)=\sum_{i=1}^{n} a_{i}-s=\left|Q_{j}\right|-s,
$$

that is,

$$
\operatorname{reg}(S / I)=\operatorname{end}(S / I)=\max \left\{\operatorname{end}\left(S / Q_{j}\right): 1 \leq j \leq r\right\}=m(I)
$$


Now let $\mathrm{ht}(I)<s$. It is harmless to assume that $\Lambda(I)=\left\{x_{1}, \ldots, x_{h}\right\}$ where $h=\mathrm{ht}(I)$. Then for a fixed $1 \leq j \leq k$, let $Q_{j}=\left(x_{1}^{b_{1}}, \ldots, x_{h}^{b_{h}}\right)$ for some positive integers $b_{1}, \ldots, b_{s}$. Thus $x_{h+1}, \ldots, x_{s}$ is an $S / I$-regular sequence. Let $J=I+S x_{h+1}+\cdots+S x_{s}$. Then $\operatorname{reg}(S / I)=\operatorname{reg}(S / J)$; see [9, Proposition 20.20] for instance. On the other hand, $J$ is $m-$ primary and so $\operatorname{reg}(S / J)=m(J)$ by the above $\operatorname{argument}$. Hence $\operatorname{reg}(S / I)=\operatorname{reg}(S / J)=$ $m(J)=\max \left\{\left(\left|Q_{j}\right|+(s-h)\right)-s: j=1, \ldots, k\right\}=m(I)$.

Theorem 3.3. Let I be an MHC monomial ideal and $\left|\operatorname{Ass}_{S} S / I\right| \leq 2$. Then we have $\operatorname{reg}(S / I)=m(I)$.

Proof. If $\left|\operatorname{Ass}_{S} S / I\right|=1$, then $I$ is a primary ideal and the result follows by Lemma 3.2. Now assume that $\left|\operatorname{Ass}_{S} S / I\right|=2$ and $\operatorname{Ass}_{S} S / I=\{\mathfrak{p}, \mathfrak{q}\}$. Let $I=I_{1} \cap I_{2}$ such that $I_{1}$ is p-primary, $I_{2}$ is q-primary and $h t\left(I_{1}\right)=\lambda(I)$. Note that

$m(I)=\max \left\{m\left(I_{1}\right), m\left(I_{2}\right)\right\}, \quad\left|\operatorname{Ass}_{S} S /\left(I_{1}+I_{2}\right)\right|=1 \quad$ and $\quad m\left(I_{1}+I_{2}\right)<m\left(I_{1}\right) \leq m(I)$.

Since $I_{1}+I_{2}$ is $(\mathfrak{p}+\mathfrak{q})$-primary

$$
\operatorname{reg}\left(S /\left(I_{1}+I_{2}\right)\right)=m\left(I_{1}+I_{2}\right)<m(I) .
$$

Now consider the exact sequence

$$
0 \longrightarrow S / I \longrightarrow S / I_{1} \oplus S / I_{2} \longrightarrow S /\left(I_{1}+I_{2}\right) \longrightarrow 0
$$

which implies that

$$
\begin{aligned}
\operatorname{reg}(S / I) & \leq \max \left\{\operatorname{reg}\left(S / I_{1} \oplus S / I_{2}\right): \operatorname{reg}\left(S /\left(I_{1}+I_{2}\right)\right)+1\right\} \\
& \leq \max \left\{\operatorname{reg}\left(S / I_{1}\right): \operatorname{reg}\left(S / I_{2}\right), m(I)\right\} \\
& \leq \max \left\{m\left(I_{1}\right): m\left(I_{2}\right), m(I)\right\}=m(I),
\end{aligned}
$$

where the second inequality follows from (3.1) and the third follows by Lemma 3.2. By the exact sequence (3.2),

$$
\operatorname{reg}\left(S / I_{1} \oplus S / I_{2}\right) \leq \max \left\{\operatorname{reg}(S / I): \operatorname{reg}\left(S /\left(I_{1}+I_{2}\right)\right)\right\}
$$

On the other hand,

$$
\begin{aligned}
\operatorname{reg}\left(S / I_{1} \oplus S / I_{2}\right) & =\max \left\{\operatorname{reg}\left(S / I_{1}\right): \operatorname{reg}\left(S / I_{2}\right)\right\} \\
& =\max \left\{m\left(I_{1}\right): m\left(I_{2}\right)\right\}=m(I)
\end{aligned}
$$

Thus $m(I) \leq \max \left\{\operatorname{reg}(S / I): \operatorname{reg}\left(S /\left(I_{1}+I_{2}\right)\right)\right\}$, from which it follows that $m(I) \leq$ $\operatorname{reg}(S / I)$ by (3.1). Now the result follows by (3.3).

REMARK 3.4. The following example shows that one cannot remove the MHC assumption in Theorem 3.3. Let $I=\left(x y, x z^{3}, y^{2}\right)=\left(x, y^{2}\right) \cap\left(y, z^{3}\right)$. Then $I$ is not MHC and $m(I)=2<3=\operatorname{reg}(S / I)$. 
REMARK 3.5. This example shows that our assumptions on $\left|\operatorname{Ass}_{S} S / I\right|$ cannot be reduced. Let $I=\left(x^{2} y, y^{2}, z^{3}\right)=\left(x, y^{2}\right) \cap\left(y, z^{3}\right) \cap\left(x^{2}, y^{2}, z\right)$. Then $I$ is MHC but $m(I)=$ $2<3=\operatorname{reg}(S / I)\left(\right.$ since $\left.\left|\operatorname{Ass}_{S} S / I\right|=3\right)$.

The behaviour of regularity with nonzero divisors is well known, that is,

$$
\operatorname{reg}(S /(I+w S))=\operatorname{reg}(S / I)+\operatorname{deg}(w)-1,
$$

where $w$ is a nonzero divisor of $S / I$. In the following we find the regularity of powers of $I+w S$ for a certain nonzero divisor $w$.

Proposition 3.6. Let I be a monomial ideal that satisfies the MHC and $w$ be an indeterminate with $w \notin \Lambda(I)$. Let $J=I+w^{d} S$ for sufficiently large $d>\operatorname{reg}(S / I)$. Then $\operatorname{reg}\left(S / J^{n}\right) \leq \operatorname{reg}(S / I)+n d-1$ for all $n \geq 1$.

Proof. Let $v:=w^{d}$. Note that by (1.1), there exists a constant integer $e$ such that for all $n \geq 1, \operatorname{reg}\left(S / I^{n}\right) \leq n d(I)+e$. In the case $n=2$,

$$
J^{2}=I^{2}+v^{2} S+v I=\left(I^{2}+v^{2} S+I\right) \cap\left(I^{2}+v^{2} S+v S\right)=\left(I+v^{2} S\right) \cap\left(I^{2}+v S\right) .
$$

Thus $m\left(J^{2}\right) \leq \max \left\{m(I)+2 d-1, m\left(I^{2}\right)+d-1\right\}$ by Lemma 2.8(iv). Hence

$$
\begin{aligned}
m\left(J^{2}\right) & \leq \max \{m(I)+2 d-1: 2 d(I)+e+d-1\} \\
& \leq \max \{\operatorname{reg}(S / I)+2 d-1: 2 d(I)+e+d-1\} \\
& \leq \max \{\operatorname{reg}(S / I)+2 d-1: 2 \operatorname{reg}(S / I)+e+d-1\},
\end{aligned}
$$

where the third inequality is due to $d(I) \leq \operatorname{reg}(S / I)$. Note that $\operatorname{reg}(S / I)$ and $e$ are constant and if we choose $v$ so that $d \geq \operatorname{reg}(S / I)+e$, then the maximum becomes $\operatorname{reg}(S / I)+2 d-1$. Thus $m\left(J^{2}\right) \leq \operatorname{reg}(S / I)+2 d-1$.

Similarly for $n \geq 2, J^{n}=\bigcap_{\substack{a+b=n+1 \\ a, b \geq 1}}\left(I^{a}+v^{b} S\right)$ and

$$
\begin{aligned}
m\left(J^{n}\right) \leq & \max \left\{m(I)+n d-1: m\left(I^{2}\right)+(n-1) d-1, \ldots, m\left(I^{n}\right)+d-1\right\} \\
= & \max \left\{\operatorname{reg}(S / I)+n d-1: \operatorname{reg}\left(S / I^{2}\right)+(n-1) d-1, \ldots, \operatorname{reg}\left(S / I^{n}\right)+d-1\right\} \\
\leq & \max \{\operatorname{reg}(S / I)+n d-1: 2 d(I)+e+(n-1) d-1, \ldots, n d(I)+e+d-1\} \\
\leq & \max \{\operatorname{reg}(S / I)+n d-1: 2 \operatorname{reg}(S / I)+e+(n-1) d-1, \ldots, \\
& \quad n \operatorname{reg}(S / I)+e+d-1\} \\
\leq & \operatorname{reg}(S / I)+\max \{n d-1: \operatorname{reg}(S / I)+e+(n-1) d-1, \ldots, \\
& n \operatorname{reg}(S / I)+e+d-1\} \\
= & \operatorname{reg}(S / I)+d-1+\max \{(n-1) d: \operatorname{reg}(S / I) \\
& +e+(n-2) d, \ldots, n \operatorname{reg}(S / I)+e\} \\
\leq & \operatorname{reg}(S / I)+n d-1 .
\end{aligned}
$$

Since $d \geq \operatorname{reg}(I)+e \geq \operatorname{reg}(S / I)+e$, we have $m\left(J^{n}\right) \leq \operatorname{reg}(S / I)+n d-1=m(I)+$ $n d-1$. On the other hand, $J$ is MHC as can be seen from the proof of Lemma 2.8(iv). 
TABLE 1. General representation of generations of $I$.

\begin{tabular}{|c|c|c|c|}
\hline$x_{11}^{a_{11}}$ & $x_{12}^{a_{12}}$ & $\cdots$ & $x_{1 t}^{a_{1 t}}$ \\
\hline$x_{21}^{a_{21}}$ & $x_{22}^{a_{22}}$ & $\cdots$ & $x_{2 t}^{a_{2 t}}$ \\
\hline$\cdots$ & $\cdots$ & $\cdots$ & $\cdots$ \\
\hline$x_{r 1}^{a_{r 1}}$ & $x_{r 2}^{a_{r 2}}$ & $\cdots$ & $x_{r t}^{a_{r t}}$ \\
\hline
\end{tabular}

Thus $J^{n}$ is MHC for all $n \geq 1$ by Proposition 2.10. Now the assertion follows from Theorem 3.3.

REMARK 3.7. It is well known that if we fix some order $>$ on $I$ and then compute the initial ideal of $I$, we have $\operatorname{reg}(I) \leq \operatorname{reg}\left(\operatorname{in}_{>} I\right)$. That is, the regularity of in $_{>} I$ provides an upper bound for the regularity of $I$. So it seems natural to pose the following questions.

Question 3.8. Which orders $>$ on $I$ will make in $>I$ to be MHC?

Question 3.9. Assume that $I$ is MHC. Then how can one compute $m\left(I^{n}\right)$ ?

To give more insight into the above questions, consider the following example. Let $R=\mathbb{Q}[x, y, z]$ with order $x>z>y$ and $I=\left(x^{2}, x y-z^{2}, y^{2} z^{2}\right)$. Then we have in $>I=$ $\left(z^{2}, x^{2}, x y^{3}\right)=\left(z^{2}, x\right) \cap\left(z^{2}, x^{2}, y^{3}\right)$. Thus $\operatorname{Ass}\left(S / \operatorname{in}_{>} I\right)=\{(x, z),(x, y, z)\}$. Hence $\operatorname{in}_{>} I$ is MHC and, for all $n \geq 1, m\left(\mathrm{in}_{>} I^{n}\right) \leq 7 n$.

\section{Appendix A. An algorithm for the computation of $m(I)$}

Assume that $I$ is a monomial ideal of $S$ with $\mu(I)=r$ and $\lambda(I)=t$. Let $G(I)=$ $\left\{u_{1}, \ldots, u_{r}\right\}$ and let $x_{i_{1}}^{a_{1}} \cdots x_{i_{t}}^{a_{t}}=\operatorname{lcm}\left(u_{1}, \ldots, u_{r}\right)$ for some $a_{i}>0$ for each $1 \leq i \leq t$. One may use the algorithmic method described in Remark 2.2 to find a primary decomposition of $I$. We have implemented this technique in Java to find $m(I)$ for a monomial ideal $I$. We may write $u_{j}=x_{i_{1}}^{a_{j 1}} \ldots x_{i_{t}}^{a_{j t}}$ for some integers $a_{j 1}, \ldots, a_{j t}$. Thus we may denote $u_{j}$ by a $t$-tuple vector $\left(a_{j 1}, \ldots, a_{j t}\right)$. We record this information, as the input of our algorithm, in Table 1.

Note that we may denote $x_{j n}^{a_{j n}}$ by the $t$-tuple $(0, \ldots, \underbrace{a_{j n}}_{n \text {th place }}, \ldots, 0)$. Thus we may replace Table 1 by Table 2 .

To find a presentation of $I$ as an intersection of pure power ideals, one should consider all possible $r$-tuples $\left(v_{1}, \ldots, v_{r}\right)$ such that $v_{j}$ is a pure power divisor of $u_{j}$, that is, $v_{j}=x_{j n}^{a_{j n}}$ for some $1 \leq j \leq r$. Since $v_{j}$ is a pure power of $t$ indeterminates, we may denote the minimal generator of the ideal $\mathfrak{p}=\left(v_{1}, \ldots, v_{r}\right)$ by a $t$-tuple vector $b=\left(b_{1}, \ldots, b_{t}\right)$ in which $b_{n}$ is a pure power of $x_{i n}$. If $b_{n}=0$ for some $1 \leq n \leq t$, then $\operatorname{ht}(\mathfrak{p}) \neq t$. Thus to find $m(I)$ we need to obtain the maximum value of $\alpha(\mathfrak{p})$ where $v_{1} \cdots v_{t} \neq 0$. In other words one should pass through all possible $t^{r}$ paths from the first row of Table 2 to the $r$ th row such that only one cell in each row is crossed at each step. 
TABLE 2. Representation of generators of $I$ by vectors.

\begin{tabular}{|c|c|c|c|}
\hline$a_{11} 0 \cdots 0$ & $0 a_{12} 0 \cdots 0$ & $\cdots$ & $0 \cdots 0 a_{1 t}$ \\
\hline$a_{21} 0 \cdots 0$ & $0 a_{22} 0 \cdots 0$ & $\cdots$ & $0 \cdots 0 a_{2 t}$ \\
\hline$\ldots$ & $\ldots$ & $\cdots$ & $\ldots$ \\
\hline$a_{r 1} 0 \cdots 0$ & $0 a_{r 2} 0 \cdots 0$ & $\cdots$ & $0 \cdots 0 a_{r t}$ \\
\hline
\end{tabular}

Set $m=0$. At the end of each path, we find the vector $b$. If $b$ is nonzero, we save it and then decide whether to keep it or not. As a matter of fact, this vector will be kept only if it is not a multiple of any of the vectors already found. Further, if either of the previously obtained vectors, say $b^{\prime}$, is a multiple of this vector, then we remove $b^{\prime}$. Finally, we set $m=\max (m:|b|-\mathrm{ht}(b))$.

The following pseudocode summarises the main iteration of this algorithm:

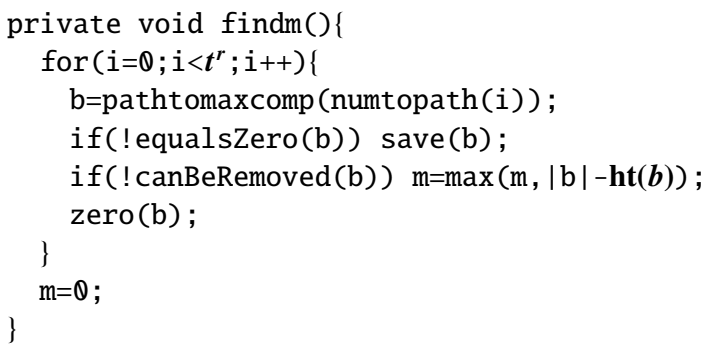

Note that numtopath will assign to each number between 1 and $t^{r}$ a path in Table 2. In Example A.1 we have shown three paths in italics.

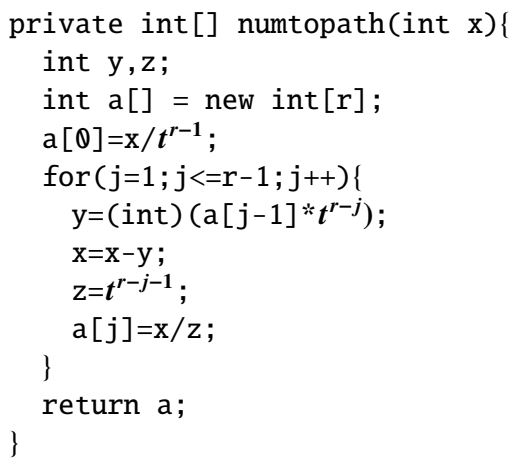

Further pathtomaxcomp will assign a $t$-tuple vector to each path. For the sake of simplicity, the way that this method performs is demonstrated for the path which is shown in italics in Table 3.

First, $b=(0,0,0)$. Following the first node in the path, $b=(2,0,0)$. Continuing the second node, $b$ is still $b=(2,0,0)$. Note that two nodes $c$ and $d$ (which are 
TABLE 3. Application of pathtomaxcomp to obtain the path which is shown in italics.

\begin{tabular}{|lllllll|lll|}
\hline 2 & 0 & 0 & 0 & 1 & 0 & 0 & 0 & 0 \\
\hline 2 & 0 & 0 & 0 & 0 & 0 & 0 & 0 & 2 \\
\hline 0 & 0 & 0 & 0 & 2 & 0 & 0 & 0 & 0 \\
\hline 0 & 0 & 0 & 0 & 1 & 0 & 0 & 0 & 3 \\
\hline
\end{tabular}

TABLE 4. Vector representation of $I$.

\begin{tabular}{|llll|lll|lll|}
\hline 2 & 0 & 0 & 0 & 1 & 0 & 0 & 0 & 0 \\
\hline 2 & 0 & 0 & 0 & 0 & 0 & 0 & 0 & 2 \\
\hline 0 & 0 & 0 & 0 & 2 & 0 & 0 & 0 & 0 \\
\hline 0 & 0 & 0 & 0 & 1 & 0 & 0 & 0 & 2 \\
\hline
\end{tabular}

essentially two vectors of size $t$ ) in each path will operate to each other by the following rule:

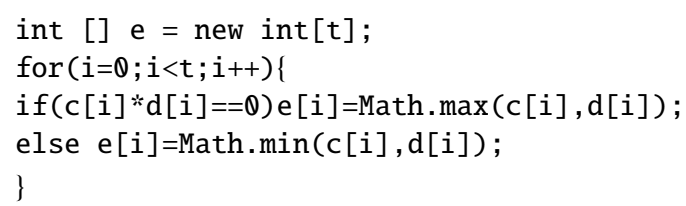

Then, following the third node, $b=(2,2,0)$. Finally, we finish this path with $b=(2,2,3)$.

Two further methods that we used in the main iteration are sketched in the following pseudocode:

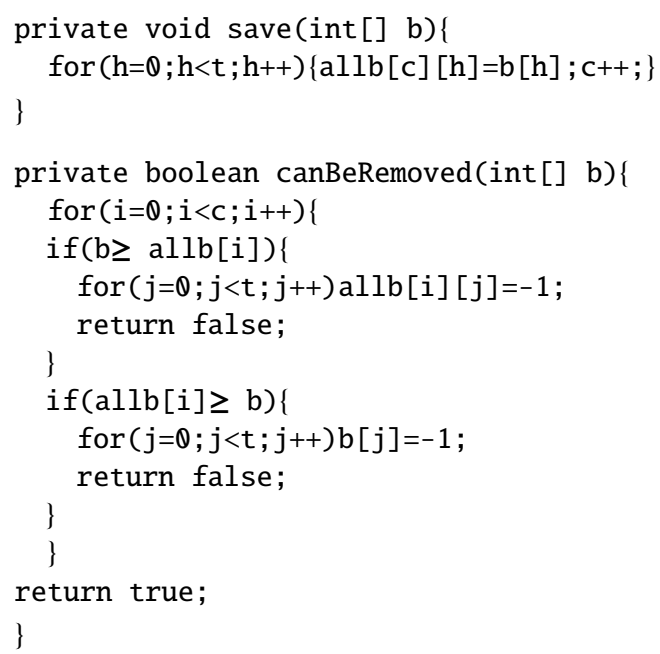


Example A.1. Let $I=\left(x_{1}^{2} x_{2}, x_{1}^{2} x_{3}^{2}, x_{2}^{2}, x_{2} x_{3}^{2}\right)$. Then Table 2 for $G(I)$ becomes Table 4 . Now consider the following three paths among all $t^{r}=3^{4}=81$ possible paths.

\begin{tabular}{|c|c|c|c|c|c|c|c|c|}
\hline 200 & 10 & 000 & 200 & 010 & 000 & 200 & $\begin{array}{llll}0 & 1 & 0\end{array}$ & \\
\hline 200 & 00 & 02 & 200 & 000 & & 200 & 000 & 02 \\
\hline 00 & & 0 & 0 & 02 & & ) & 02 & 0 \\
\hline 000 & 10 & 0002 & $\begin{array}{llll}0 & 0 & 0\end{array}$ & 010 & $\begin{array}{llll}0 & 0 & 2\end{array}$ & 0000 & 010 & 002 \\
\hline
\end{tabular}

For path $(1), b=(2,0,0)$ and so $m=(2+0+0)-1=1$. For path $(2), b=(2,1,0)$ and so $m=(2+1+0)-2=1$. For path $(3), b=(2,2,2)$ and so $m=(2+2+2)-3=3$. As may easily be seen, the value of $m(I)$ does not increase along other remaining paths. Hence $m(I)=3=\operatorname{reg}(S / I)$.

Remark A.2. During this project we became aware that Bermejo et al. [2] produced a library for doing computations with monomial ideals in SINGULAR. In particular, they implemented several methods for constructing the irredundant irreducible decomposition of a monomial ideal.

\section{Acknowledgements}

The authors are grateful to Professors S. Zarzuela, I. Bermejo and J. Alvarez for fruitful discussions and useful comments regarding this paper. Furthermore, the authors would like to thank the anonymous referee for very useful comments and suggestions.

\section{References}

[1] A. Aramova, K. Crona and E. De Negri, 'Bigeneric initial ideals, diagonal subalgebras and bigraded Hilbert functions', J. Pure Appl. Algebra 150 (2000), 215-235.

[2] I. Bermejo, E. Garcia-Llorente and Ph. Gimenez, 'monomialideal.lib. A SINGULAR library for computing with monomial ideals', SINGULAR 3.1.1 2010.

[3] K. Borna, 'On linear resolution of powers of an ideal', Osaka J. Math. 46(4) (2009), 1047-1058.

[4] M. P. Brodmann and R. Y. Sharp, Local Cohomology: an Algebraic Introduction with Geometric Applications, Cambridge Studies in Advanced Mathematics, 60 (Cambridge University Press, Cambridge, UK, 1998).

[5] M. Chardin, 'Powers of ideals and the cohomology of stalks and fibers of morphisms', arxiv:1009.1271.

[6] CoCoATeam, 'CoCoA: a system for doing Computations in Commutative Algebra', available at http://cocoa.dima.unige.it.

[7] A. Conca, 'Regularity jumps for powers of ideals', in: Commutative Algebra, Lecture Notes in Pure and Applied Mathematics, 244 (Chapman \& Hall/CRC, Boca Raton, FL, 2006), 21-32.

[8] S. D. Cutkosky, J. Herzog and N. V. Trung, 'Asymptotic behaviour of the Castelnuovo-Mumford regularity’, Compositio Math. 118 (1999), 243-261.

[9] D. Eisenbud, Commutative Algebra With A View Toward Algebraic Geometry, Graduate Texts in Mathematics, 150 (Springer, Berlin, 1995).

[10] J. Herzog and T. Hibi, 'The depth of powers of an ideal', J. Algebra 291 (2005), 534-550. 
[11] J. Herzog and T. Hibi, Monomial Ideals, Graduate Texts in Mathematics, 260 (Springer, Berlin, 2011).

[12] J. Herzog, T. Hibi and X. Zheng, 'Monomial ideals whose powers have a linear resolution', Math. Scand. 95(1) (2004), 23-32.

[13] L. T. Hoa and N. V. Trung, 'On the Castelnuovo-Mumford regularity and the arithmetic degree of monomial ideals', Math. Z. 229 (1998), 519-537.

[14] V. Kodiyalam, 'Asymptotic behaviour of Castelnuovo-Mumford regularity', Proc. Amer. Math. Soc. 128 (2000), 407-411.

[15] T. Römer, 'On minimal graded free resolutions', Illinois J. Math. 45(2) (2001), 1361-1376.

KEIVAN BORNA, Faculty of Mathematics and Computer Science, Kharazmi University, Tehran, Iran

and

School of Mathematics, Institute for Research in Fundamental Sciences (IPM), PO Box 19395-5746, Tehran, Iran

e-mail: borna@ipm.ir

RAHELEH JAFARI, School of Mathematics, Institute for Research in Fundamental Sciences (IPM), PO Box 19395-5746, Tehran, Iran

e-mail: rjafari@ipm.ir 